\title{
latrogenic intramural dissection of the gallbladder wall can mimic post-ERCP cholecystitis
}

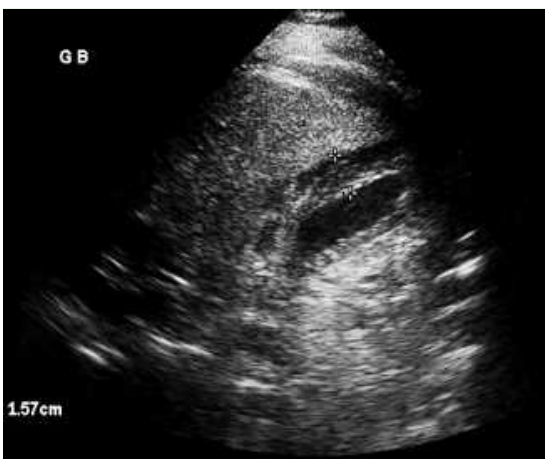

Figure 1 Ultrasonography performed 1 day after the endoscopic retrograde cholangiopancreatography (ERCP) revealed a gallbladder wall thickness greater than $15 \mathrm{~mm}$ with no pericholecystic fluid.

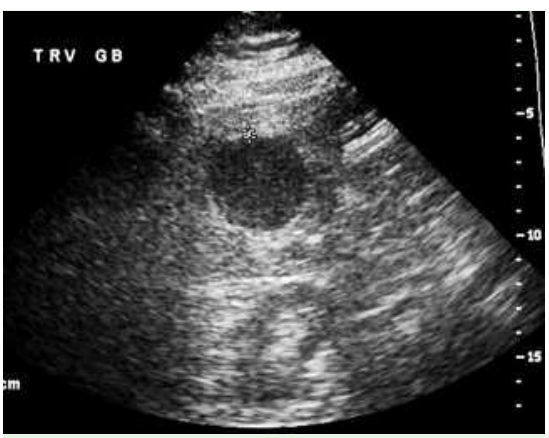

Figure 2 Ultrasonography performed 4 days before ERCP demonstrated a normal gallbladder wall thickness of $2.8 \mathrm{~mm}$.

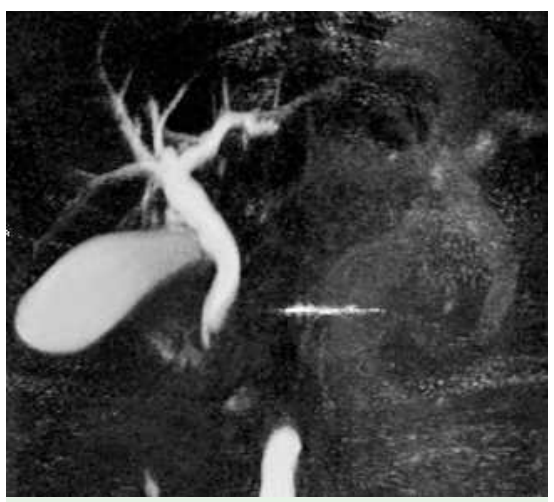

Figure 3 Magnetic resonance cholangiopancreatography performed 3 days before ERCP demonstrated a patent cystic duct and good visualization of the gallbladder.

A 28-year-old female was referred for surgical management of acute cholecystitis 1 day after endoscopic retrograde cholangiopancreatography (ERCP) and biliary
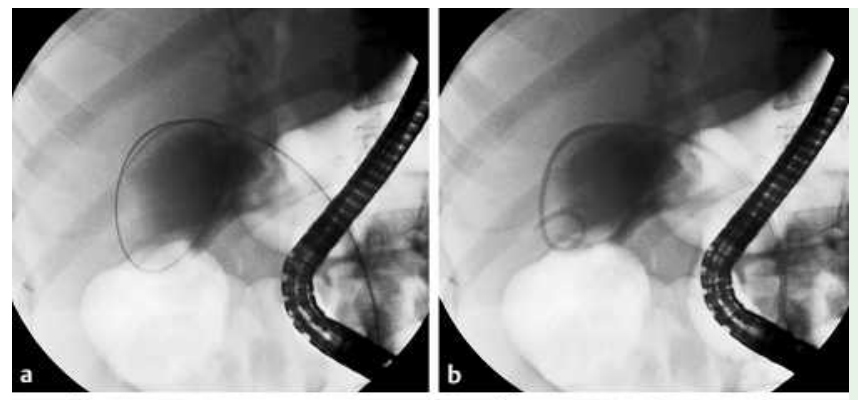

Figure 4 The initial fluoroscopic image obtained after the guide wire was placed into the gallbladder does not reveal the injury. Infiltration of contrast material into the gallbladder wall with lifting of the mucosa off the
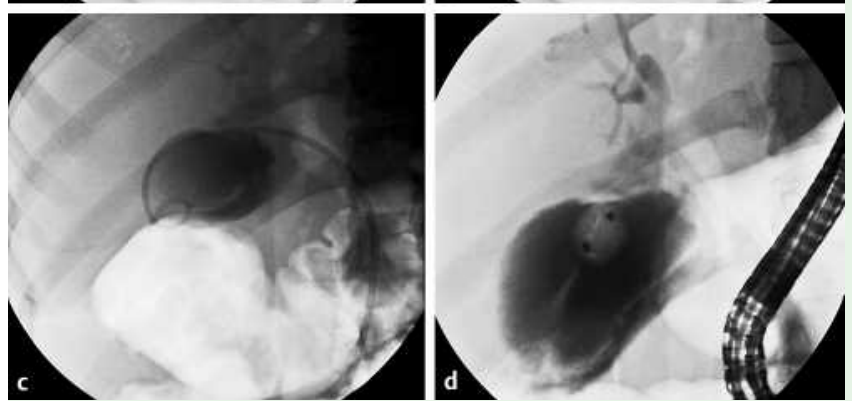
muscularis demonstrated that the guide wire and catheter caused an intramural dissection during the ERCP.
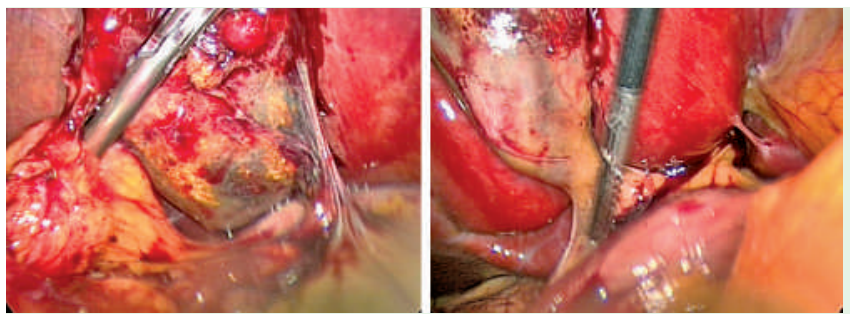

Figure 5 Laparoscopy demonstrated a grayish-blue discoloration of the gallbladder consistent with an intramural hematoma. sphincterotomy, because of the finding of a 15-mm-thick gallbladder wall on right upper quadrant ultrasonography (๑ Figure 1). Pre-ERCP ultrasonography (๑ Figure 2) and magnetic resonance cholangiopancreatography ( Figure 3 ) demonstrated a $2.8-\mathrm{mm}$ gallbladder wall and a patent cystic duct. The fluoroscopic images of the ERCP were reexamined and it was apparent that introduction of the guide wire had caused a dissection of the gallbladder wall which was visualized only after the injury had been exacerbated by injection of contrast intramurally ( $\bullet$ Figure 4). In the absence of fever, leukocytosis, a positive Murphy's sign, or pericholecystic fluid on ultrasound images, the gallbladder wall thickening was concluded to represent an iatrogenic injury. We monitored the patient with serial abdominal exams to rule out a perforation and were able to discharge her with conservative management alone. Two months later she underwent an elective laparoscopic cholecystectomy for symptoms attributed to cholecystitis. A mural hematoma was seen upon initial visualization of the gallbladder ( $\bullet$ Figure 5 ) and confirmed by histopathology.

The incidence of post-ERCP acute cholecystitis is less than $1 \%[1,2]$. The etiology has been postulated to be the presence of nonsterile contrast medium exacerbated by cystic duct obstruction and mechanical irritation [3-5]. This case represents the first reported occurrence of an intramural dissection of the gallbladder wall during ERCP. The subsequent intramural hematoma caused gallbladder wall thickening that mimicked post-ERCP cholecystitis on ultrasonography. While concurrent development of localized tenderness, fever, leukocytosis, and pericholecystic fluid on ultrasonography would strongly suggest post-ERCP cholecystitis, an isolated and sudden increase in gallbladder wall thickness after ERCP must be evaluated carefully for the possibility 
of an iatrogenic injury with the attendant risk of perforation.

Endoscopy_UCTN_Code_CPL_1AK_2AB

Endoscopy_UCTN_Code_CPL_1AK_2AC

M. E. Girotti ${ }^{1}$, N. Gupta ${ }^{2}$, B. D. Schirmer ${ }^{2}$, M. Sarti ${ }^{3}$, A. F. Choudhri ${ }^{3}$, B. Arslan ${ }^{3}$,

A. T. Schroen ${ }^{2}$

1 University of Virginia School of Medicine, Charlottesville, VA 22908, USA

2 Department of Surgery, University of Virginia Health System, Charlottesville, VA 22908, USA

3 Department of Radiology, University of Virginia Health System, Charlottesville, VA 22908, USA

\section{References}

1 Loperfido S, Angelini G, Benedetti G et al. Major early complications from diagnostic and therapeutic ERCP: a prospective multicenter study. Gastrointest Endosc 1998; 48: 1 10

2 Freeman ML, Nelson DB, Sherman $S$ et al. Complications of endoscopic biliary sphincterotomy. N Engl J Med 1996; 335: 909-918

3 Leung JW, Chung SC, Sung JY, Li MK. Acute cholecystitis after stenting of the common bile duct for obstruction secondary to pancreatic cancer. Gastrointest Endosc 1989; 35: $109-110$

4 Dolan R, Pinkas H, Brady PG. Acute cholecystitis after palliative stenting for malignant obstruction of the biliary tree. Gastrointest Endosc 1993; 39: 447-449

5 Alvarez C, Hunt K, Ashley SW, Reber HA. Emphysematous cholecystitis after ERCP. Dig Dis Sci 1994; 39: 1719-1723
Bibliography

DOI 10.1055/s-2007-966366

Endoscopy 2007; 39: E205-E206

(c) Georg Thieme Verlag KG Stuttgart · New York . ISSN 0013-726X

\section{Corresponding author}

\section{N. Gupta, MD, PhD}

University of Virginia Health System

PO Box 800300

Charlottesville

VA 22908

USA

Fax: +1-434-243-5791

naren@virginia.edu 